\title{
The Effects of Longitudinal White Matter Hyperintensity Change on Cognitive Decline and Cortical Thinning over Three Years
}

\author{
Seung Joo Kim ${ }^{1,2,+}{ }^{\oplus}$, Dong Kyun Lee ${ }^{3,+}$, Young Kyoung Jang ${ }^{1}$, Hyemin Jang ${ }^{1,4,5}$, \\ Si Eun Kim ${ }^{1,6}$, Soo Hyun Cho ${ }^{1,7}$, Jun Pyo Kim ${ }^{1,8}$, Young Hee Jung ${ }^{1,9}$, Eun-Joo Kim ${ }^{10}$, \\ Duk L. Na 1,4,5,11, Jong-Min Lee ${ }^{12}$, Sang Won Seo ${ }^{1,4,5,11,13,14}$ and Hee Jin Kim 1,4,5,11,14,* \\ 1 Department of Neurology, Samsung Medical Centre, Sungkyunkwan University School of Medicine, \\ Seoul 135-710, Korea; workcare50@gmail.com (S.J.K.); blurgroove@gmail.com (Y.K.J.); \\ hmjang57@gmail.com (H.J.); tldmsldi@naver.com (S.E.K.); k906141h@hanmail.net (S.H.C.); \\ torch0703@gmail.com (J.P.K.); neophilia1618@gmail.com (Y.H.J.); dukna@naver.com (D.L.N.); \\ sangwonseo@empal.com (S.W.S.) \\ 2 Departments of Neurology, Gyeongsang National University Changwon Hospital, Changwon 51372, Korea \\ 3 Department of Mental Health Research, National Centre for Mental Health, Seoul 04933, Korea; \\ babadk2@gmail.com \\ 4 Neuroscience Centre, Samsung Medical Centre, Seoul 135-710, Korea \\ 5 Samsung Alzheimer Research Centre, Samsung Medical Centre, Seoul 135-710, Korea \\ 6 Departments of Neurology, Inje University College of Medicine, Haeundae Paik Hospital, \\ Busan 48108, Korea \\ 7 Department of Neurology, Chonnam National University Hospital, Gwangju 501-757, Korea \\ 8 Department of Radiology and Imaging Sciences, Indiana University School of Medicine, \\ Indianapolis, IN 47405, USA \\ 9 Department of Neurology, Myongji Hospital, Hanyang University, Goyang 10475, Korea \\ 10 Department of Neurology, Pusan National University Hospital, Busan 49241, Korea; eunjookim@pusan.ac.kr \\ 11 Department of Health Sciences and Technology, Sungkyunkwan University, Seoul 135-710, Korea \\ 12 Department of Biomedical Engineering, Hanyang University, Seoul 11923, Korea; ljm@hanyang.ac.kr \\ 13 Department of Clinical Research Design and Evaluation, Sungkyunkwan University, Seoul 135-710, Korea \\ 14 Department of Digital Health, SAIHST, Sungkyunkwan University, Seoul 135-710, Korea \\ * Correspondence: evekhj@gmail.com; Tel.: +82-2-3410-6147 \\ $\dagger$ Seung Joo Kim and Dong Kyun Lee contributed equally to this work.
}

Received: 24 June 2020; Accepted: 15 August 2020; Published: 17 August 2020

\begin{abstract}
White matter hyperintensity (WMH) has been recognised as a surrogate marker of small vessel disease and is associated with cognitive impairment. We investigated the dynamic change in WMH in patients with severe WMH at baseline, and the effects of longitudinal change of WMH volume on cognitive decline and cortical thinning. Eighty-seven patients with subcortical vascular mild cognitive impairment were prospectively recruited from a single referral centre. All of the patients were followed up with annual neuropsychological tests and 3T brain magnetic resonance imaging. The WMH volume was quantified using an automated method and the cortical thickness was measured using surface-based methods. Participants were classified into WMH progression and WMH regression groups based on the delta WMH volume between the baseline and the last follow-up. To investigate the effects of longitudinal change in $\mathrm{WMH}$ volume on cognitive decline and cortical thinning, a linear mixed effects model was used. Seventy patients showed WMH progression and 17 showed WMH regression over a three-year period. The WMH progression group showed more rapid cortical thinning in widespread regions compared with the WMH regression group. However, the rate of cognitive decline in language, visuospatial function, memory and executive function, and general cognitive function was not different between the two groups. The results of this study indicated that WMH volume changes are dynamic and WMH progression is associated with more rapid cortical thinning.
\end{abstract}


Keywords: white matter hyperintensity; cortical thinning; cognitive decline; subcortical vascular mild cognitive impairment

\section{Introduction}

Subcortical white matter hyperintensities (WMHs), common findings in aging, are associated with vascular risk factors, and have been recognised as a surrogate marker of cerebral small vessel disease (SVD) [1,2]. In several cross-sectional studies, WMH volume was reportedly associated with decreased cortical thickness [3,4] and cognitive dysfunction [5]. The effects of SVD change on cognitive decline have been evaluated in several longitudinal studies. The progression of SVD or WMH was shown to be associated with cognitive decline over time [6,7]. With the recent advances in imaging techniques, WM integrity can also be quantified by measuring the mobility of the water molecules in the WM microstructure. Individuals with a lower WM integrity at baseline tended to progress to cognitive impairment more often than those with a higher WM integrity in a longitudinal study [8]. In addition, a high WMH burden at baseline led to an increased risk of rapid WMH progression over time [9]. In addition, the WMH volume changes were suggested to be dynamic, indicating that WMH may progress or regress over time $[10,11]$. According to these studies, the change in WMH volume per year was variable. The average increased $\mathrm{WMH}$ volume ranged from $0.1 \mathrm{~mL} /$ year to $2.2 \mathrm{~mL} /$ year, and the average regressed $\mathrm{WMH}$ volume ranged from $-0.02 \mathrm{~mL} /$ year to $-0.6 \mathrm{~mL} /$ year [11-15]. Some longitudinal studies suggested that WMH regression might be due to measurement errors $[11,16]$ or pathophysiologic changes after ischemic insult $[10,17]$. WMH at earlier stages may reflect altered interstitial fluid mobility and water content as "pre-visible" changes, which may be reversible; however, in later stages, demyelination and axonal damage may occur, which may be irreversible [17].

The effects of SVD or WMH change on cortical thickness and cognition have been simultaneously evaluated in only a few studies $[18,19]$. In a previous study, the SVD progression group showed more rapid cognitive decline and brain atrophy compared with the SVD stable group [19]. In another study, the WMH regression or stable group showed improved memory function and decreased brain atrophy compared with the WMH progression group [18]. However, these studies were performed among patients with varying degrees of SVD. Knowledge is limited regarding the effects of WMH change on cognitive decline or cortical thinning in patients with severe SVD at baseline, such as subcortical vascular mild cognitive impairment (svMCI).

We hypothesised that the WMH volume in svMCI patients would show dynamic changes over time. Our previous longitudinal study showed that svMCI patients show overall cortical thinning and cognitive decline over three years [20]. We evaluated whether the patients in the WMH progression group would show more rapid cortical thinning and cognitive decline compared with patients in the WMH regression group.

\section{Methods}

\subsection{Participants}

In the present study, 87 patients with svMCI were prospectively recruited from the Memory Disorder Clinic at Samsung Medical Centre from July 2006 to August 2011, who annually underwent high-resolution 3.0-Tesla brain magnetic resonance imaging (MRI) and neuropsychological tests for three years. The patients had to meet the following criteria to be diagnosed with svMCI: (1) subjective memory complaints made by the patient or caregiver; (2) an objective cognitive impairment below -1.0 standard deviations (SD) of age- and education-matched norms in at least one cognitive domain on neuropsychological tests; (3) normal activities of daily living as determined by a clinician; (4) subcortical vascular features such as a focal neurological symptoms or signs; and (5) severe WMH on T2-weighted and fluid attenuated inversion recovery (FLAIR) images defined by periventricular 
$\mathrm{WMH} \geq 10 \mathrm{~mm}$ and deep WMH $\geq 25 \mathrm{~mm}$, which was modified from the Fazekas ischemia criteria [21]. Patients received clinical interviews and neurological examinations. All of the patients underwent a $\left[{ }^{11} \mathrm{C}\right]$ Pittsburgh compound B (PiB) positron emission tomography (PET) scan. Patients with territorial cerebral infarction, brain tumour, and vascular malformation were excluded based on the brain MRI.

During the three-year follow-up period, the mean duration of follow-up was 33 months and patients repeated neuropsychological tests and brain MRI four times. After 87 patients completed the baseline neuropsychological test, 87 patients completed the first-year follow-up, 77 patients completed the second-year follow-up, and 65 patients completed the third-year follow-up. After 87 patients completed the baseline brain MRI, 87 patients completed the first-year follow-up, 77 patients completed the second-year follow-up, and 61 patients completed the third-year follow-up.

\subsection{Acquisition of MR Images}

We acquired standardized 3D T1 turbo field echo images and 3D FLAIR images from all of the patients using the same 3.0 T MRI scanner (Philips 3.0 T Achieva; Philips Health care). Briefly, the 3D T1 MR images were set to the following parameters: sagittal slice thickness, $1.0 \mathrm{~mm}$, over contiguous slices with 50\% overlap; no gap; a repetition time of $9.9 \mathrm{~ms}$; an echo time of $4.6 \mathrm{~ms}$; a flip angle of $8^{\circ}$; and a matrix size of $240 \times 240$ pixels reconstructed to $480 \times 480$ over a field of view of $240 \mathrm{~mm}$. The 3D FLAIR image parameters included the following: axial slice thickness of $2 \mathrm{~mm}$; no gap; repetition time of $11,000 \mathrm{~ms}$; echo time of $125 \mathrm{~ms}$; flip angle of $90^{\circ}$; and matrix size of $512 \times 512$ pixels.

\subsection{Measurement of Longitudinal WMH Volume}

First, the baseline WMH volume was quantified using an automated algorithm, as previously described [22]. The baseline WMH candidate regions on the FLAIR images were extracted by applying a classification method and morphological operation to 3D T1-weighted images. To extract the baseline $\mathrm{WMH}$, a threshold method was applied to the FLAIR images within the WMH candidate regions. Although the threshold value was selected considering the range of image intensities, segmented results could contain false-positive or false-negative regions, depending on the extent of WMH. The rate of agreement between two neurologists was $92.3 \%$. If the results contained an error, the threshold value was reselected through visual inspection by two raters, and they reached a consensus in the case of discrepancy.

Second, the longitudinal WMH volume change was measured by evaluating the difference between baseline and follow-up FLAIR images [23]. Each follow-up brain image was transformed separately into a baseline brain space using affine transform. Because the shooting time of each longitudinal image was different, each image intensity was converted to a z-score. Then, the longitudinal WMH volume change was measured using the difference in z-score between baseline and follow-up FLAIR images.

Finally, patients were divided into the WMH progression (baseline WMH volume $<$ last follow-up $\mathrm{WMH}$ volume) and WMH regression (baseline WMH volume > last follow-up WMH volume) groups based on the WMH volume change.

\subsection{Acquisition of $\left[{ }^{11} \mathrm{C}\right]$ PiB PET and Data Analysis}

To measure the amyloid burden, all of the patients with svMCI underwent $\left[{ }^{11} \mathrm{C}\right] \mathrm{PiB}$ PET scans with a Discovery Ste PET/computed tomography scanner (GE Medical Systems, Milwaukee, WI, USA). $\left[{ }^{11} \mathrm{C}\right]$ PiB PET scanning was performed using 3D scanning mode examining 35 slices of $4.25-\mathrm{mm}$ thickness, spanning the entire brain. The CT scan was performed for attenuation correction, and 60-min and 30-min emission static PET scans were initiated after the injection of a mean dose of $420 \mathrm{MBq}\left[{ }^{11} \mathrm{C}\right] \mathrm{PiB}$. The specific radioactivity of $\left[{ }^{11} \mathrm{C}\right] \mathrm{PiB}$ for patients was $>1500 \mathrm{Ci} / \mathrm{mmol}$ at the time of administration, and the radiochemical yield was $>35 \%$. All of the PET studies had $>95 \%$ in the radiochemical purity of the tracer.

The PiB PET images were co-registered to individual MRIs, which were normalized to a T1 MR image template. 
To measure the $\mathrm{PiB}$ retention, the cerebellar grey matter was used as a reference region [24]. We selected 28 cortical volumes of interest from the left and right hemispheres using the annotated anatomical labelling (AAL) atlas. The cerebral cortical volumes of interest consisted of the bilateral frontal cortices, posterior cingulate gyri, parietal, lateral temporal, and occipital areas. The regional cerebral cortical uptake ratios were calculated by dividing each cortical volume of interest uptake ratio by the mean uptake of the cerebellar cortex (cerebellum crus 1 and crus 2). The global PiB standardised uptake value ratio (SUVR) was calculated from the volume-weighted average uptake ratio of the bilateral 28 cerebral cortical volumes of interest. Patients were defined as PiB positive if their global $\mathrm{PiB}$ retention ratio was more than $2.0 \mathrm{SD}$ ( $\mathrm{PiB}$ retention ratio $>1.5$ ) from the mean of the healthy controls [25].

\subsection{Cortical Thickness Analyses}

The process of the cortical thickness measurement was described in previous longitudinal studies [20,26]. T1-weighted images were processed using the standard Montreal Neurological Institute anatomical pipeline. The native MRI images were registered to a standardized stereotaxic space through a linear transformation [27], and the N3 algorithm was used to correct the images for intensity-based non-uniformities caused by non-homogeneities in the magnetic field [28]. Then, the registered and corrected images underwent segmentation into white matter, grey matter, and nonbrain tissue using the Intensity-Normalized Stereotaxic Environment for Classification of Tissues algorithm [29]. The surfaces of the inner and outer cortex were automatically extracted using the Constrained Laplacian-Based Automated Segmentation with Proximities algorithm [30]. Although cortical thickness was defined as the Euclidean distance between the linked vertices of these areas [30], the values were calculated in the native brain spaces, rather than using the Talairach space, given the existing limitations in linear stereotaxic normalization. The MR volumes in the native space were transformed into the stereotaxic space with a linear transformation matrix, and the reconstructing process was performed using the inverse transformation matrix [31]. Subsequently, the thickness value was spatially normalized using surface-based two-dimensional registration with a sphere-to-sphere warping algorithm, meaning that the vertices of each subject were non-linearly registered to a standard surface template [32]. We used intracranial volume (ICV) as a covariate to control for brain volume in the statistical analyses. For the global and lobar regional analyses, the data that were previously manually categorised to lobes with a high inter-rater reliability [31] were registered to the template. Finally, the cortical surface of each subject was divided into frontal, temporal, parietal, and occipital lobes via the automated processes.

\subsection{Neuropsychological Tests}

Patients underwent annual neuropsychological tests using the Seoul Neuropsychological Screening Battery (SNSB) [33,34]. SNSB consists of several tests for language and related functions, visuospatial functions, verbal and visual memory, and executive functions. The scores of each domain in the SNSB were considered abnormal when they were lower than -1.0 SD for age- and education-matched norms. In this study, domain composite scores were derived by summing the selective tests scores for each subdomain [35]. The language and related functions domain score were derived from the raw score of Korean-version of the Boston Naming Test (0-60). The Rey Complex Figure Test (RCFT) copy score was used for the visuospatial functions domain (0-36). The memory domain was calculated from the sum of the immediate-recall score, 20-min delayed-recall score, and a recognition score from the Seoul Verbal Leaning Test (three learning/free recall trials of 12 words, a 20-min delayed-recall trial for these 12 items, and a recognition test) and RCFT (0-168). The executive functions domain was calculated from the sum of the phonemic and semantic Controlled Oral Word Association Test and the Stroop Colour Test (colour reading of 112 items during a 2-min period; 0-173). All of the patients also performed the Clinical Dementia Rating Scale Sum of Boxes (0-18) and the Korean version of the Mini-Mental State Examination (0-30). All of the tests were performed by experienced staff and supervised by board-certified clinical neuropsychologists. 


\subsection{Statistical Analyses}

To compare the demographic variables between the WMH progression and WMH regression groups, the Student's $t$-test was performed for continuous variables and the Chi-square test or Fisher's exact test was performed for categorical variables.

To evaluate the effect of the longitudinal change of the $\mathrm{WMH}$ volume on the longitudinal cognitive decline and cortical thinning, a linear mixed effects model was performed. We included subjects and slope as random components, and baseline age, sex, time, group, PiB PET SUVR [25], ICV (for analysis of the cortical thickness), and the interaction between groups and time as the fixed components, because these factors could affect the WMH volume. We also performed a sub-analysis after excluding the amyloid PET positive svMCI patients. For the statistical analyses, SPSS 21.0 was used (SPSS Inc., Chicago, IL, USA).

To evaluate the topography of the cortical thinning associated with a change in WMH volume, MATLAB-based toolbox was used (available online through the University of Chicago: https:// galton.uchicago.edu/faculty/InMemoriam/worsley/research/surfstat). To blur each cortical thickness map, a full-width half-maximum diffusion smoothing of $20 \mathrm{~mm}$ was used, resulting in an increased signal-to-noise ratio and statistical power [36]. Linear mixed effects models were used vertex-by-vertex to analyse the localised differences and the statistical map of cortical thickness on the surface model. Baseline age, sex, time, group, ICV, PiB PET SUVR, and the interaction between groups and time were entered as the fixed effects, and the subject was entered as a random effect. Because multiple comparisons were performed for 81,924 vertices, an uncorrected $p$-value of $<0.01$ was considered statistically significant.

\subsection{Standard Protocol Approval, Registration, and Patient Consent}

The Institutional Review Board of the Samsung Medical Centre approved the study and all of the patients signed informed consent (IRB no. 2008-08-018).

\section{Results}

\subsection{Demographics}

The demographics and clinical characteristics at baseline are summarised in Table 1. Among the 87 patients, 70 showed a progression of WMH volume and 17 showed a regression of WMH volume over a three-year period. Baseline demographics, vascular risk factors, SVD burden, and antiplatelet use were not significantly different between the WMH progression and WMH regression groups. The total $\mathrm{WMH}$ volume at baseline was $40.98 \pm 29.13 \mathrm{~mL}$ in the $\mathrm{WMH}$ progression group and $38.97 \pm 24.72 \mathrm{~mL}$ in the WMH regression group. In the WMH progression group, the mean WMH volume increased $0.29 \mathrm{~mL}$ per year $(p<0.001)$ and in the $\mathrm{WMH}$ regression group, WMH volume decreased $0.06 \mathrm{~mL}$ per year $(p=0.006)$.

Table 1. Demographics and clinical characteristics of patients in the WMH progression and WMH regression groups at baseline.

\begin{tabular}{lccc}
\hline & WMH Progression $(\boldsymbol{n}=\mathbf{7 0})$ & WMH Regression $(\boldsymbol{n}=\mathbf{1 7})$ & $\boldsymbol{p}$-Value \\
\hline Age $($ mean $\pm \mathrm{SD})$, years & $72.1 \pm 6.5$ & $71.8 \pm 8.7$ & 0.158 \\
Female, $n(\%)$ & $39(55.7)$ & $12(70.6)$ & 0.264 \\
Education (mean \pm SD), years & $9.9 \pm 5.5$ & $8.1 \pm 5.0$ & 0.229 \\
Cardiovascular risk factors, $n(\%)$ & & & \\
$\quad$ BMI $\left(\mathrm{kg} / \mathrm{m}^{2}\right)$ & $25.05 \pm 4.39$ & $22.97 \pm 3.47$ & 0.073 \\
$\quad$ Hypertension & $54(77.1)$ & $13(76.5)$ & 1.000 \\
$\quad$ Diabetes mellitus & $15(21.4)$ & $5(29.4)$ & 0.526 \\
$\quad$ Hyperlipidaemia & $21(30)$ & $3(17.6)$ & 0.378 \\
APOE4, $n(\%)$ & $17(24.3)$ & $6(35.3)$ & 0.370 \\
Anti-platelet agent, $n(\%)$ & $62(88.6)$ & $14(82.4)$ & 0.443 \\
\hline
\end{tabular}


Table 1. Cont.

\begin{tabular}{|c|c|c|c|}
\hline & WMH Progression $(n=70)$ & WMH Regression $(n=17)$ & $p$-Value \\
\hline Anti-coagulant agent, $n(\%)$ & $1(1.4)$ & $0(0.0)$ & 0.014 \\
\hline \multicolumn{4}{|l|}{ Baseline SVD markers } \\
\hline $\mathrm{WMH}($ mean $\pm \mathrm{SD}), \mathrm{mL}$ & $40.98 \pm 29.13$ & $38.97 \pm 24.72$ & 0.794 \\
\hline Microbleed (mean \pm SD),$n$ & $5.2 \pm 12.2$ & $3.6 \pm 6.3$ & 0.648 \\
\hline Lacunae (mean $\pm \mathrm{SD}), n$ & $6.3 \pm 7.3$ & $4.3 \pm 4.1$ & 0.254 \\
\hline \multicolumn{4}{|l|}{ PiB PET } \\
\hline PiB SUVR & $1.48 \pm 0.40$ & $1.53 \pm 0.38$ & 0.711 \\
\hline PiB positive (SUVR > 1.5), $n(\%)$ & $18 / 63(28.6)$ & $4 / 10(40)$ & 0.476 \\
\hline Cortical thickness (mean $\pm \mathrm{SD}$ ), mm & $2.84 \pm 0.16$ & $2.77 \pm 0.18$ & 0.130 \\
\hline \multicolumn{4}{|l|}{ Cognition } \\
\hline Language & $40.20 \pm 9.08$ & $36.24 \pm 12.22$ & 0.223 \\
\hline Visuospatial function & $27.07 \pm 7.97$ & $27.56 \pm 7.79$ & 0.820 \\
\hline Memory & $75.76 \pm 17.54$ & $76.88 \pm 21.60$ & 0.845 \\
\hline Executive function & $97.40 \pm 35.74$ & $87.88 \pm 26.80$ & 0.230 \\
\hline MMSE & $25.91 \pm 3.13$ & $24.82 \pm 3.84$ & 0.290 \\
\hline CDR-SB & $1.29 \pm 1.07$ & $1.74 \pm 1.64$ & 0.295 \\
\hline Mean follow-up duration & $34.01 \pm 10.13$ & $29.06 \pm 13.48$ & 0.095 \\
\hline
\end{tabular}

\subsection{Longitudinal Change of Cortical Thickness}

In all of the svMCI patients, the patients in the WMH progression group showed more rapid cortical thinning in the frontal, temporal, parietal, and occipital lobes than the patients in the WMH regression group (Table 2). A sub-analysis including only PiB negative svMCI patients also showed that patients in the WMH progression group had more rapid cortical thinning in the frontal, temporal, and parietal lobes than patients in the WMH regression group (Table 2). We further calculated the delta WMH for each year and ran correlation analyses with this value. However, delta WMH was not correlated with longitudinal cortical thinning (Supplementary Table S1).

Table 2. Longitudinal change of cortical thickness in the white matter hyperintensity (WMH) progression group compared with the WMH regression group.

\begin{tabular}{ccccc}
\hline & \multicolumn{2}{c}{ All svMCI $(\boldsymbol{n}=\mathbf{8 7})$} & \multicolumn{2}{c}{ PiB Negative svMCI $(\boldsymbol{n}=\mathbf{5 1})$} \\
\cline { 2 - 5 } & B (SE) & $\boldsymbol{p}$-Value & B (SE) & $\boldsymbol{p}$-Value \\
\hline Global thickness & $-0.034(0.012)$ & 0.006 & $-0.027(0.012)$ & 0.030 \\
Frontal lobe & $-0.041(0.013)$ & 0.002 & $-0.028(0.013)$ & 0.033 \\
Temporal lobe & $-0.040(0.016)$ & 0.014 & $-0.035(0.015)$ & 0.024 \\
Parietal lobe & $-0.034(0.012)$ & 0.005 & $-0.032(0.012)$ & 0.009 \\
Occipital lobe & $-0.025(0.011)$ & 0.029 & $-0.019(0.014)$ & 0.173 \\
\hline
\end{tabular}

Abbreviations: WMH— white matter hyperintensity; SE-standard error. A linear mixed effects model was performed after adjusting for baseline age, sex, time, group, intracranial volume, Pittsburgh compound B standardised uptake value ratio, and the interaction between the groups and time.

A statistical map showed that in all svMCI patients, the WMH progression group had more rapid cortical thinning in the bilateral frontal and lateral temporal regions than the WMH regression group (Figure 1A). A sub-analysis including only PiB negative svMCI patients also showed that the WMH progression group had more rapid cortical thinning in the right frontal and bilateral temporal regions than the WMH regression group (Figure 1B). 


\section{A All svMCl patients}
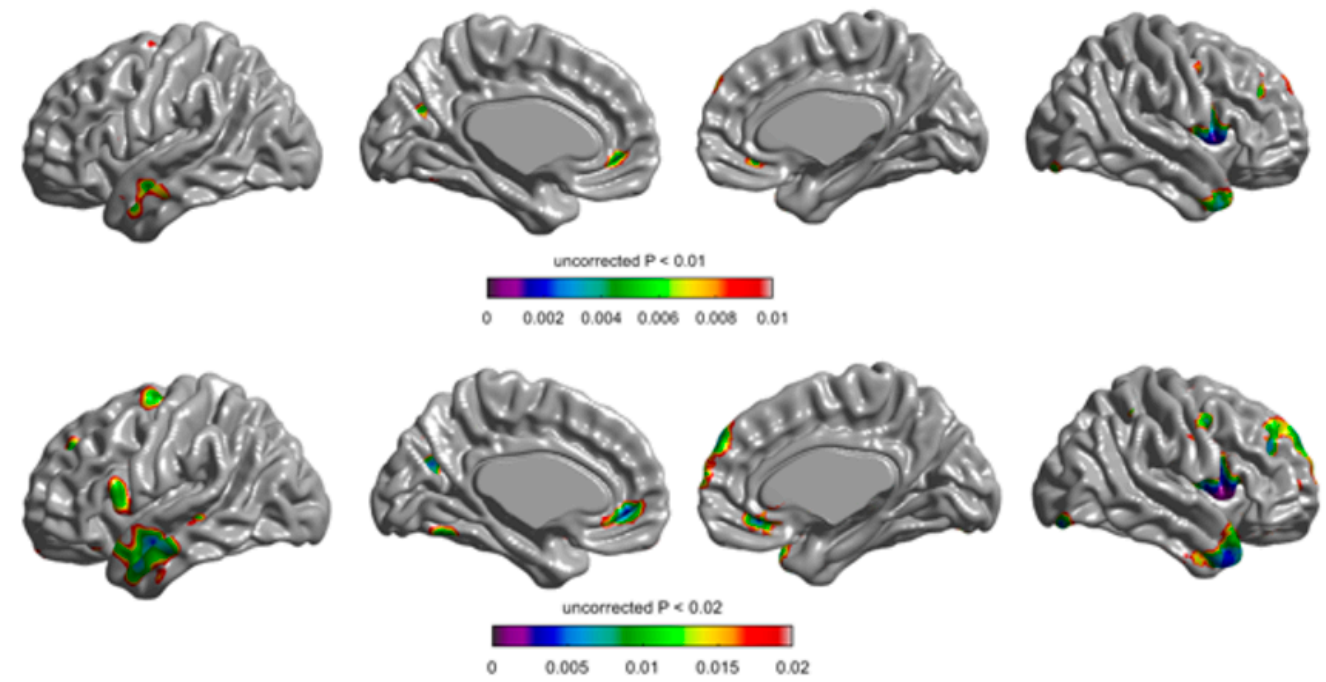

\section{B PiB negative svMCl patients}
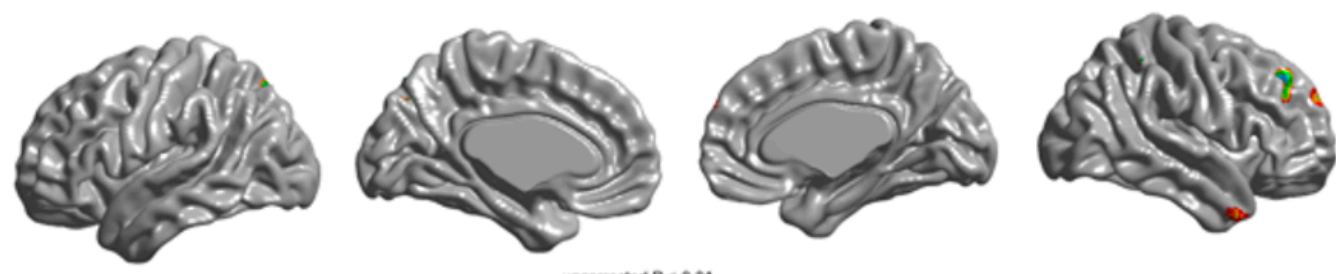

uncorrected $P<0.01$

$\begin{array}{llllll}0 & 0.002 & 0.004 & 0.006 & 0.008 & 0.01\end{array}$
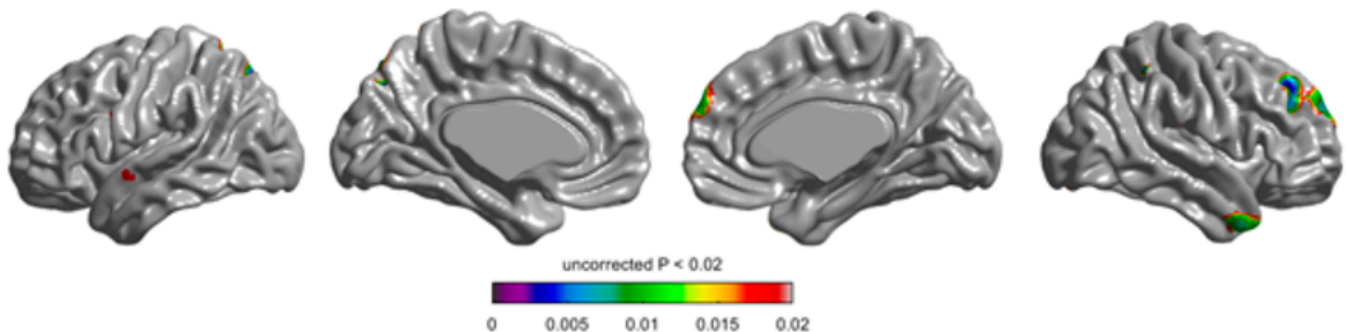

Figure 1. Longitudinal change of cortical thickness in the white matter hyperintensity (WMH) progression group compared with the WMH regression group in all of the subcortical vascular mild cognitive impairment (svMCI) patients (A) and in PiB negative svMCI patients (B). Compared with the WMH progression group, the WMH regression group demonstrated more rapid cortical thinning in the coloured areas. Baseline age, sex, time, group, intracranial volume, Pittsburgh compound B standardised uptake value ratio, and the interaction between the groups and time were entered as fixed effects, and subject was entered as a random effect (uncorrected $p<0.01$ in the upper row and $p<0.02$ in the lower row).

\subsection{Longitudinal Change of Cognition}

In all of the svMCI patients, the rates of cognitive decline in language, visuospatial function, memory, executive function, MMSE, and CDR-SB were not different between the WMH progression and WMH regression groups (Table 3). Similarly, in the PiB negative svMCI patients, the rates of cognitive decline in language, visuospatial function, memory, executive function, MMSE, and CDR-SB were not different between the WMH progression and WMH regression groups (Table 3). Further analysis showed that there was no significant difference in the rate of cognitive decline between the WMH fast progression group $(n=35)$ and the WMH slow progression group $(n=35)$. 
Table 3. Longitudinal change of cognitive function in the WMH progression group compared with the $\mathrm{WMH}$ regression group.

\begin{tabular}{lcccc}
\hline & \multicolumn{2}{c}{ All svMCI $(n=87)$} & \multicolumn{2}{c}{ PiB negative svMCI $(n=51)$} \\
\cline { 2 - 5 } & B (SE) & $p$-Value & B (SE) & $p$-Value \\
\hline Cognition & & & & \\
$\quad$ Language & $1.076(0.699)$ & 0.129 & $0.869(0.693)$ & 0.217 \\
$\quad$ Visuospatial function & $-0.277(0.712)$ & 0.699 & $0.949(0.831)$ & 0.260 \\
$\quad$ Memory & $1.873(2.189)$ & 0.395 & $2.792(2.442)$ & 0.259 \\
Executive function & $2.348(3.396)$ & 0.492 & $0.564(3.912)$ & 0.886 \\
MMSE & $0.419(0.386)$ & 0.281 & $0.301(0.402)$ & 0.457 \\
CDR-SB & $-0.034(0.226)$ & 0.880 & $-0.0665(0.239)$ & 0.782 \\
\hline
\end{tabular}

Abbreviations: WMH—white matter hyperintensity; SE-standard error; MMSE-mini-mental status examination; CDR-SB - clinical dementia rating-sum of boxes. A linear mixed effects model was performed after adjusting for baseline age, sex, time, group, Pittsburgh compound B standardised uptake value ratio, and the interaction between the groups and time.

\section{Discussion}

In the present study, dynamic changes in WMH were observed in svMCI patients over a three-year period. The major findings of the study were the following: (1) among the 87 svMCI patients, 17 patients $(19.5 \%)$ showed a regression in WMH volume over a three-year period; (2) compared with the WMH regression group, the WMH progression group showed more rapid cortical thinning in the frontal and lateral temporal regions; and (3) a significant difference in the rate of cognitive decline was not observed between the WMH regression and WMH progression groups. Collectively, we suggest that the change in $\mathrm{WMH}$ volume was dynamic in svMCI patients, and WMH progression was associated with more rapid cortical thinning.

Our first major finding in the study was that $19.5 \%$ of svMCI patients showed significant WMH regression, while others showed WMH progression over a three-year period. This finding was in agreement with a recent report in which SVD markers demonstrated dynamic changes, as $20.3 \%$ of patients showed SVD regression over 8.7 years [19]. In another study of AD patients with varying degrees of SVD, the authors reported that the WMH volume was dynamic because it shrank, grew, or remained stable over time [14]. Despite recent published reports, the present study is unique because all of the svMCI patients in the cohort had a severe level of WMH volume at baseline, which corresponds to Fazekas III. Several hypotheses for WMH regression are possible $[11,14,18]$. First, the baseline WMH may have been due to transient inflammatory processes, which might resolve over time. Transient fluid-related WM damage might resolve before permanent axonal injury or demyelination [17]. Second, reversible ischemic injury might heal over time $[18,19]$. Third, pathophysiological changes such as blood-brain barrier disruption and the clearance of fluid after a small subcortical stroke could also be a possible underlying mechanism [14,37].

Significant differences were not observed in the demographics or baseline vascular risk factors between the WMH progression and WMH regression groups, which was in agreement with previous studies [18,19], although in one study, the body mass index in the WMH progression group was significantly higher than in the WMH regression group [19].

Second, the WMH progression group was associated with more rapid cortical thinning than the WMH regression group over a three-year period. Specifically, the WMH progression group showed rapid cortical thinning in the bilateral frontal and lateral temporal regions, which was in agreement with our previous study showing that lacunae progression was associated with cortical thinning in the frontal regions [26]. A sub-analysis, which included only amyloid negative svMCI patients, also showed that patients in the WMH progression group had more rapid cortical thinning in the frontal, temporal, and parietal lobes than patients in the WMH regression group. To date, chronic ischemia in the brain leads to demyelination and axonal loss, which results in WMH [21]. In addition, microglial and endothelial activation in the interstitial tissue of the brain leads to neuronal loss, resulting in 
cortical thinning [38]. For these reasons, WMH accompanies cortical thinning [39]. However, in the early phase of ischemia, interstitial fluid in the oedematous lesion may be reversed and the transient inflammatory process might heal $[17,18]$, thereby reducing $\mathrm{WMH}$ and protecting against neuronal loss and cortical thinning $[18,19]$. Thus, these pathomechanisms might explain the dynamic change of WMH and its relationship with cortical thinning. In our additional analysis, we could not yield a significant correlation between the delta WMH for each year and cortical thinning. This might be due to a small variation in the delta WMH.

Finally, our data showed that in svMCI patients, the rate of cognitive decline was not significantly different between the WMH progression and WMH regression groups. A sub-analysis including only $\mathrm{PiB}$ negative svMCI patients also showed that the rate of cognitive decline was not different between the WMH progression and WMH regression groups. In contrast to our results, there are studies that have shown that the $\mathrm{WMH}$ progression group had a more rapid cognitive decline than the WMH regression or stable group $[18,19]$. The discrepancy might be explained by the different degrees of WMH burden in each study cohort. Patients in previous studies had varying degrees of WMH burden; however, the patients in this study had a severe WMH burden at baseline. Therefore, although the $\mathrm{WMH}$ regression group showed an overall net regression in WMH volume, severe WMH at baseline may have already led to a vicious cascade of network disruption, resulting in significant cognitive decline [19]. It is also possible that we might not have found a significant difference in the rate of cognitive decline because of the relatively small sample size.

The strength of the current study was that patients with severe WMH were prospectively recruited, and cortical thickness and cognitive function were repeatedly measured. However, the present study had several limitations. First, the sample size was relatively small. Thus, statistical differences in the rate of cognitive decline between the WMH progression and WMH regression groups, or correlation between the delta WMH for each year and cortical thinning may not have been found. The results should be interpreted with caution, and need to be confirmed in studies with a larger sample size. Second, three years of follow-up is not sufficient to observe biological changes associated with WMH dynamics, and studies with longer follow-up period are needed. Finally, whether strict control of vascular risk factors and a healthy lifestyle may mitigate $\mathrm{WMH}$ progression should be investigated in future studies. Despite these limitations, the results of the present study highlight the importance of dynamic WMH volume changes, which affect cortical thickness.

\section{Conclusions}

In conclusion, we found that the change in WMH volume was dynamic, as some svMCI patients showed WMH regression, while others showed WMH progression. WMH progression was associated with more rapid cortical thinning in the frontal and temporal areas. Our results suggest that identifying the dynamic change in WMH volume in svMCI patients could be valuable for predicting the rate of neurodegeneration. Further investigations are needed to assess whether strict risk factor control slows down WMH progression and protects cortical thinning or cognitive decline.

Supplementary Materials: The following are available online at http://www.mdpi.com/2077-0383/9/8/2663/s1: Table S1, Correlation between delta WMH and longitudinal change of cortical thickness.

Author Contributions: Study concept and design, S.J.K., D.K.L., D.L.N., J.-M.L., S.W.S., and H.J.K. Acquisition, analysis, or interpretation of data, Y.K.J., H.J., S.E.K., S.H.C., J.P.K., Y.H.J., and E.-J.K. drafting of the manuscript, S.W.S. and H.J.K. All authors have read and agreed to the published version of the manuscript.

Funding: This research received no external funding.

Acknowledgments: This research was supported by a National Research Foundation (NRF) of Korea grant funded by the Korea government (MSIP; NRF-2018R1A1A3A04079255); the Korean Health Technology R\&D Project, Ministry of Health and Welfare, Republic of Korea (HI18C0335, HI18C1629); the MSIT (Ministry of Science and ICT), Korea, under the ICT Creative Consilience program (IITP-2020-0-01821) supervised by the IITP (Institute for Information \& communications Technology Planning \& Evaluation); and a grant of the Korea Health Technology R\&D Project through the Korea Health Industry Development Institute (KHIDI), funded by the Ministry of Health and Welfare, Republic of Korea (HI19C1132). 
Conflicts of Interest: The authors declare that they have no competing interest.

\section{References}

1. DeCarli, C.; Murphy, D.; Tranh, M.; Grady, C.L.; Haxby, J.V.; Gillette, J.A.; Salerno, J.A.; Gonzales-Aviles, A.; Honvitz, B.; Rapoport, S.I.; et al. The effect of white matter hyperintensity volume on brain structure, cognitive performance, and cerebral metabolism of glucose in 51 healthy adults. Neurology 1995, 45, 2077-2084. [CrossRef] [PubMed]

2. Wardlaw, J.M.; Smith, E.E.; Biessels, G.J.; Cordonnier, C.; Fazekas, F.; Frayne, R.; Lindley, R.I.; O’Brien, J.; Barkhof, F.; Benavente, O.R.; et al. Neuroimaging standards for research into small vessel disease and its contribution to ageing and neurodegeneration. Lancet Neurol. 2013, 12, 822-838. [CrossRef]

3. Kim, H.J.; Ye, B.S.; Yoon, C.W.; Noh, Y.; Kim, G.H.; Cho, H.; Jeon, S.; Lee, J.M.; Kim, J.-H.; Seong, J.-K.; et al. Cortical thickness and hippocampal shape in pure vascular mild cognitive impairment and dementia of subcortical type. Eur. J. Neurol. 2014, 21, 744-751. [CrossRef] [PubMed]

4. Seo, S.W.; Ahn, J.; Yoon, U.; Im, K.; Lee, J.-M.; Kim, S.T.; Ahn, H.-J.; Chin, J.; Jeong, Y.; Na, D.L. Cortical Thinning in Vascular Mild Cognitive Impairment and Vascular Dementia of Subcortical Type. J. Neuroimaging 2010, 20, 37-45. [CrossRef] [PubMed]

5. Prins, N.D.; Scheltens, P. White matter hyperintensities, cognitive impairment and dementia: An update. Nat. Rev. Neurol. 2015, 11, 157-165. [CrossRef] [PubMed]

6. Van Dijk, E.J.; Prins, N.D.; Vrooman, H.A.; Hofman, A.; Koudstaal, P.J.; Breteler, M.M.B. Progression of Cerebral Small Vessel Disease in Relation to Risk Factors and Cognitive Consequences. Stroke 2008, 39, 2712-2719. [CrossRef]

7. Maillard, P.; Carmichael, O.; Fletcher, E.; Reed, B.; Mungas, D.; DeCarli, C. Coevolution of white matter hyperintensities and cognition in the elderly. Neurology 2012, 79, 442-448. [CrossRef]

8. Zhuang, L.; Sachdev, P.S.; Trollor, J.N.; Kochan, N.A.; Reppermund, S.; Brodaty, H.; Wen, W. Microstructural white matter changes in cognitively normal individuals at risk of amnestic MCI. Neurology 2012, 79, 748-754. [CrossRef]

9. Wardlaw, J.M.; Doubal, F.N.; Valdes-Hernandez, M.; Wang, X.; Chappell, F.M.; Shuler, K.; Armitage, P.A.; Carpenter, T.C.; Dennis, M.S. Blood-brain barrier permeability and long-term clinical and imaging outcomes in cerebral small vessel disease. Stroke 2012, 44, 525-527. [CrossRef]

10. Wardlaw, J.M.; Chappell, F.M.; Hernández, M.D.C.V.; Makin, S.D.; Staals, J.; Shuler, K.; Thrippleton, M.J.; Armitage, P.A.; Muñoz-Maniega, S.; Heye, A.K.; et al. White matter hyperintensity reduction and outcomes after minor stroke. Neurology 2017, 89, 1003-1010. [CrossRef]

11. Van Leijsen, E.M.; De Leeuw, F.-E.; Tuladhar, A.M. Disease progression and regression in sporadic small vessel disease-insights from neuroimaging. Clin. Sci. 2017, 131, 1191-1206. [CrossRef] [PubMed]

12. Cho, A.-H.; Kim, H.-R.; Kim, W.; Yang, D.W. White Matter Hyperintensity in Ischemic Stroke Patients: It May Regress Over Time. J. Stroke 2015, 17, 60-66. [CrossRef] [PubMed]

13. Kloppenborg, R.P.; Nederkoorn, P.J.; Geerlings, M.I.; Berg, E.V.D. Presence and progression of white matter hyperintensities and cognition: A meta-analysis. Neurology 2014, 82, 2127-2138. [CrossRef] [PubMed]

14. Ramirez, J.; McNeely, A.A.; Berezuk, C.; Gao, F.; Black, S.E. Dynamic Progression of White Matter Hyperintensities in Alzheimer's Disease and Normal Aging: Results from the Sunnybrook Dementia Study. Front. Aging Neurosci. 2016, 8, 62. [CrossRef] [PubMed]

15. Benjamin, P.; Zeestraten, E.; Lambert, C.; Chis-Ster, I.; Williams, O.; Lawrence, A.J.; Patel, B.; MacKinnon, A.D.; Barrick, T.R.; Markus, H.S. Progression of MRI markers in cerebral small vessel disease: Sample size considerations for clinical trials. Br. J. Pharmacol. 2015, 36, 228-240. [CrossRef]

16. Schmidt, R.; Ropele, S.; Enzinger, C.; Petrovic, K.; Smith, S.M.; Schmidt, H.; Matthews, P.M.; Fazekas, F. White matter lesion progression, brain atrophy, and cognitive decline: The Austrian stroke prevention study. Ann. Neurol. 2005, 58, 610-616. [CrossRef]

17. Wardlaw, J.M.; Hernández, M.C.V.; Muñoz-Maniega, S. What are White Matter Hyperintensities Made of? J. Am. Hear. Assoc. 2015, 4, 1140. [CrossRef]

18. Al-Janabi, O.; Bauer, C.E.; Goldstein, L.B.; Murphy, R.R.; Bahrani, A.A.; Smith, C.D.; Wilcock, D.M.; Gold, B.T.; Jicha, G.A. White Matter Hyperintensity Regression: Comparison of Brain Atrophy and Cognitive Profiles with Progression and Stable Groups. Brain Sci. 2019, 9, 170. [CrossRef] 
19. Van Leijsen, E.M.; Bergkamp, M.; Van Uden, I.W.; Cooijmans, S.; Ghafoorian, M.; Van Der Holst, H.M.; Norris, D.G.; Kessels, R.P.; Platel, B.; Tuladhar, A.M.; et al. Cognitive consequences of regression of cerebral small vessel disease. Eur. Stroke J. 2018, 4, 85-89. [CrossRef]

20. Lee, J.; Seo, S.W.; Yang, J.-J.; Jang, Y.K.; Lee, J.S.; Kim, Y.J.; Chin, J.; Lee, J.M.; Kim, S.T.; Lee, K.-H.; et al. Longitudinal cortical thinning and cognitive decline in patients with early- versus late-stage subcortical vascular mild cognitive impairment. Eur. J. Neurol. 2017, 25, 326-333. [CrossRef]

21. Fazekas, F.; Kleinert, R.; Offenbacher, H.; Schmidt, R.; Kleinert, G.; Payer, F.; Radner, H.; Lechner, H. Pathologic correlates of incidental MRI white matter signal hyperintensities. Neurology 1993, 43, 1683. [CrossRef] [PubMed]

22. Jeon, S.; Yoon, U.; Park, J.-S.; Seo, S.W.; Kim, J.-H.; Kim, S.T.; Kim, S.I.; Na, D.L.; Lee, J.-M. Fully automated pipeline for quantification and localization of white matter hyperintensity in brain magnetic resonance image. Int. J. Imaging Syst. Technol. 2011, 21, 193-200. [CrossRef]

23. Carmichael, O.; Schwarz, C.G.; Drucker, D.; Fletcher, E.; Harvey, D.; Beckett, L.A.; Jack, C.R.; Weiner, M.; DeCarli, C.; Initiative, A.D.N. Longitudinal Changes in White Matter Disease and Cognition in the First Year of the Alzheimer Disease Neuroimaging Initiative. Arch. Neurol. 2010, 67, 1370-1378. [CrossRef] [PubMed]

24. Rowe, C.C.; Ellis, K.; Rimajova, M.; Bourgeat, P.; Pike, K.E.; Jones, G.R.; Fripp, J.; Tochon-Danguy, H.; Morandeau, L.; O'Keefe, G.; et al. Amyloid imaging results from the Australian Imaging, Biomarkers and Lifestyle (AIBL) study of aging. Neurobiol. Aging 2010, 31, 1275-1283. [CrossRef] [PubMed]

25. Lee, J.H.; Kim, S.H.; Kim, G.H.; Seo, S.W.; Park, H.K.; Oh, S.J.; Kim, J.S.; Cheong, H.-K.; Na, D.L. Identification of pure subcortical vascular dementia using 11C-Pittsburgh compound B. Neurology 2011, 77, 18-25. [CrossRef]

26. Kim, H.J.; Yang, J.J.; Kwon, H.; Kim, C.; Lee, J.M.; Chun, P.; Kim, Y.J.; Jung, N.-Y.; Chin, J.; Kim, S.; et al. Relative impact of amyloid- $\beta$, lacunes, and downstream imaging markers on cognitive trajectories. Brain 2016, 139, 2516-2527. [CrossRef]

27. Collins, D.L.; Neelin, P.; Peters, T.M.; Evans, A.C. Automatic 3D Intersubject Registration of MR Volumetric Data in Standardized Talairach Space. J. Comput. Assist. Tomogr. 1994, 18, 192-205. [CrossRef]

28. Sled, J.G.; Zijdenbos, A.; Evans, A. A nonparametric method for automatic correction of intensity nonuniformity in MRI data. IEEE Trans. Med Imaging 1998, 17, 87-97. [CrossRef]

29. Zijdenbos, A.; Forghani, R.; Evans, A. Automatic "pipeline" analysis of 3-D MRI data for clinical trials: Application to multiple sclerosis. IEEE Trans. Med Imaging 2002, 21, 1280-1291. [CrossRef]

30. Kim, J.S.; Singh, V.; Lee, J.K.; Lerch, J.; Ad-Dab’Bagh, Y.; Macdonald, D.; Lee, J.M.; Kim, S.I.; Evans, A.C. Automated 3-D extraction and evaluation of the inner and outer cortical surfaces using a Laplacian map and partial volume effect classification. NeuroImage 2005, 27, 210-221. [CrossRef]

31. Im, K.; Lee, J.-M.; Lee, J.; Shin, Y.W.; Kim, I.Y.; Kwon, J.S.; Kim, S.I. Gender difference analysis of cortical thickness in healthy young adults with surface-based methods. NeuroImage 2006, 31, 31-38. [CrossRef] [PubMed]

32. Lyttelton, O.; Boucher, M.; Robbins, S.; Evans, A.C. An unbiased iterative group registration template for cortical surface analysis. NeuroImage 2007, 34, 1535-1544. [CrossRef] [PubMed]

33. Ahn, H.-J.; Chin, J.; Park, A.; Lee, B.H.; Suh, M.K.; Seo, S.W.; Na, D.L. Seoul Neuropsychological Screening Battery-Dementia Version (SNSB-D): A Useful Tool for Assessing and Monitoring Cognitive Impairments in Dementia Patients. J. Korean Med Sci. 2010, 25, 1071-1076. [CrossRef] [PubMed]

34. Kang, S.H.; Park, Y.H.; Lee, D.; Kim, J.P.; Chin, J.; Ahn, Y.; Park, S.B.; Kim, H.J.; Jang, H.; Jung, Y.H.; et al. The Cortical Neuroanatomy Related to Specific Neuropsychological Deficits in Alzheimer's Continuum. Dement. Neurocognitive Disord. 2019, 18, 77-95. [CrossRef]

35. Kim, H.J.; Park, S.; Cho, H.; Jang, Y.K.; Lee, J.S.; Jang, H.; Kim, Y.; Kim, K.W.; Ryu, Y.H.; Choi, J.Y.; et al. Assessment of Extent and Role of Tau in Subcortical Vascular Cognitive Impairment Using 18F-AV1451 Positron Emission Tomography Imaging. JAMA Neurol. 2018, 75, 999-1007. [CrossRef]

36. Scheltens, P.; Barkhof, F.; Leys, D.; Pruvo, J.; Nauta, J.; Vermersch, P.; Steinling, M.; Valk, J. A semiquantative rating scale for the assessment of signal hyperintensities on magnetic resonance imaging. J. Neurol. Sci. 1993, 114, 7-12. [CrossRef]

37. Wardlaw, J.M.; Sandercock, P.; Dennis, M.; Starr, J.; Kalimo, H. Is Breakdown of the Blood-Brain Barrier Responsible for Lacunar Stroke, Leukoaraiosis, and Dementia? Stroke 2003, 34, 806-812. [CrossRef] 
38. Tomimoto, H.; Akiguchi, I.; Suenaga, T.; Nishimura, M.; Wakita, H.; Nakamura, S.; Kimura, J. Alterations of the Blood-Brain Barrier and Glial Cells in White-Matter Lesions in Cerebrovascular and Alzheimer's Disease Patients. Stroke 1996, 27, 2069-2074. [CrossRef]

39. Jang, H.; Kwon, H.; Yang, J.-J.; Hong, J.; Kim, Y.; Kim, K.W.; Lee, J.S.; Jang, Y.K.; Kim, S.T.; Lee, K.H.; et al. Correlations between Gray Matter and White Matter Degeneration in Pure Alzheimer's Disease, Pure Subcortical Vascular Dementia, and Mixed Dementia. Sci. Rep. 2017, 7, 9541. [CrossRef]

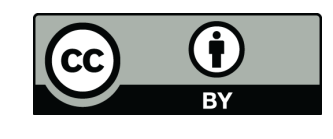

(C) 2020 by the authors. Licensee MDPI, Basel, Switzerland. This article is an open access article distributed under the terms and conditions of the Creative Commons Attribution (CC BY) license (http://creativecommons.org/licenses/by/4.0/). 